\title{
Neural processing of working memory in adults with ADHD in a visuospatial change detection task with distractors
}

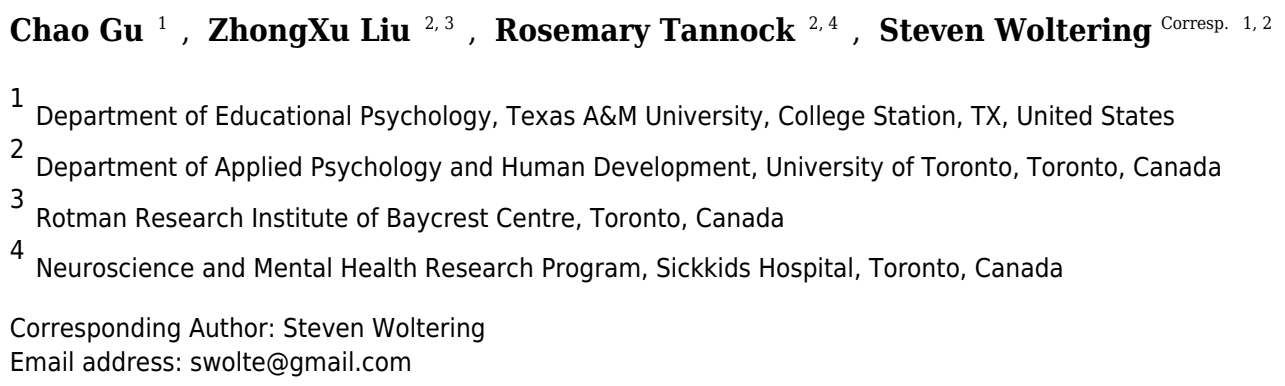

Individuals with Attention-Deficit Hyperactivity-Disorder (ADHD) are often characterized by deficits in working memory (WM), which manifest in academic, professional, and mental health difficulties. To better understand the underlying mechanisms of these presumed WM deficits, we compared adults with ADHD to their peers on behavioral and neural indices of WM. We used a visuospatial change detection task with distractors which was designed to assess the brain's ability to effectively filter out distractors from WM, in addition to testing for effects of WM load. Twenty-seven unmedicated adults with ADHD were compared to 27 matched peers on event-related potential (ERP) measures of WM, i.e., the Contralateral Delay Activity (CDA). Despite severe impairments in everyday life functioning, findings showed no difference in deficits in behavioral tests of working memory for adults with ADHD compared to their peers. Interestingly, there were differences in neural activity between individuals with ADHD and their peers showing that the CDA of individuals with ADHD did not distinguish between high, distractor, and low memory load conditions. These data suggest, in the face of comparable behavioral performance, a difference in neural processing efficiency, wherein the brains of individuals with ADHD may not be as selective in the allocation of neural resources to perform a WM task. 
Neural Processing of Working Memory in Adults with ADHD in a Visuospatial Change Detection Task with Distractors

Chao Gu${ }^{1}$, Zhong-Xu Liu ${ }^{2,3}$, Rosemary Tannock ${ }^{2,4}, \&$ Steven Woltering ${ }^{1,2, ~ *}$

${ }^{1}$ Department of Educational Psychology, Texas A\&M University, USA

${ }^{2}$ Department of applied Psychology and Human Development, University of Toronto, Canada

${ }^{3}$ Rotman Research Institute of Baycrest Centre, Toronto, Canada.

${ }^{4}$ Neurosciences and Mental Health Research Program, Sickkids Hospital, Toronto, Canada

* Corresponding Author

Steven Woltering

Department of Educational Psychology, Texas A\&M University

Email: swolte@tamu.edu

Phone: 979.862 .8973 


\section{Abstract}

5 Individuals with Attention-Deficit Hyperactivity-Disorder (ADHD) are often characterized by

6 deficits in working memory (WM), which manifest in academic, professional, and mental health

7 difficulties. To better understand the underlying mechanisms of these presumed WM deficits, we

8 compared adults with ADHD to their peers on behavioral and neural indices of WM. We used a

9 visuospatial change detection task with distractors which was designed to assess the brain's

10 ability to effectively filter out distractors from WM, in addition to testing for effects of WM load.

11 Twenty-seven unmedicated adults with ADHD were compared to 27 matched peers on event-

12 related potential (ERP) measures of WM, i.e., the Contralateral Delay Activity (CDA). Despite severe impairments in everyday life functioning, findings showed no difference in behavioral tests of working memory for adults with ADHD compared to their peers. Interestingly, there were differences in neural activity between individuals with ADHD and their peers showing that the CDA of individuals with ADHD did not distinguish between high, distractor, and low memory load conditions. These data suggest, in the face of comparable behavioral performance, a difference in neural processing efficiency, wherein the brains of individuals with ADHD may not be as selective in the allocation of neural resources to perform a WM task. 


\section{Introduction}

Attention-Deficit/Hyperactivity Disorder (ADHD) is a prevalent mental health disorder characterized by persistent behavioral symptoms of inattention and hyperactivity/impulsivity, which can severely impede educational, occupational and health outcomes (de Graaf et al., 2008; Polanczyk \& Jensen, 2008; Uchida et al., 2018). Visuospatial Working Memory (WM) impairments, as a sub-component of working memory (Baddeley, 2010), are impaired in individuals with ADHD compared to the general population, and can contribute to, or exaggerate, overall functional impairments (Hervey, Epstein, \& Curry, 2004; Martinussen et al., 2005; Chamberlain et al., 2011; Fried et al., 2016).

WM refers to a limited-capacity, multi-component cognitive system that is required to

34 keep relevant information in mind for a few seconds while performing complex tasks such as reasoning and comprehension (Baddeley, 2010; Miyake \& Shah, 1999). Evidence of poor WM performance has generally been assumed to reflect a smaller capacity to store information, i.e.,

37 individuals with poor WM hold fewer items in mind compared to those with good WM (Bunge, 38 Klingberg, Jacobsen, \& Gabrieli, 2000; Cowan, 2001). However, researchers have pointed to attention as a control process, which regulates access to WM, to be influential as well (Awh \&

40 Vogel, 2006; Cowan et al., 2005; Shipstead, Lindsey, Marshall, \& Engle, 2014). In fact, this line 41 of research suggests that storage capacity may be an outcome rather than a mechanism of WM, 42 and that filtering efficiency (the ability to inhibit irrelevant information from entering into WM) 43 may be the primary neurocognitive mechanism that helps control it (Vogel, McCollough, \& 44 Machizawa, 2005; Vogel \& Machizawa, 2004; Kuo, Strokes, \& Nobre, 2012). 

adults with ADHD and their peers using a visuospatial change detection working memory task while electroencephalography (EEG) was recorded. Similar to Vogel et al., (2004), we measured the Contralateral Delay Activity (CDA) which is an event-related potential (ERP; averaged EEG waves locked to an event) measured at posterior sites during the retention period. The CDA's amplitude increases reliably with the number of items to be remembered in the task's different memory load conditions; and has been shown to reach a limit at an individual's WM capacity

52 (Luria et al., 2016). The difference in amplitude between low and high load conditions has been

53

found to predict behavioral (i.e., neuropsychological) measures of storage capacity (Vogel et al., 2005; McCollough, Machizawa, \& Vogel, 2007; Diamantopoulou, Poom, Klaver, \& Talsma, 2011; Kundu, Sutterer, Emrich, \& Postle, 2013). We will refer to this measure as CDA- $\Delta$. The sensitivity of the CDA waveform to load can also be used to determine an index of filtering efficiency: in other words, whether irrelevant items unnecessarily consume WM capacity. In the distractor version of this task, participants are asked to remember the colors of squares ( 2 squares in the low load and 4 in high load) in an array but to ignore the circles in a distractor condition ( 2 squares +2 circles). If the CDA of the distractor condition $(2$ circles +2 squares) resembles the low load condition (2 squares), we can conclude that participants have effectively filtered out the irrelevant circles and excluded them from storage. Conversely, if the CDA for the distractor condition resembles that of the high load condition (4 squares), participants may have failed to efficiently filter out the distractors. Vogel et al., (2004; 2005) have shown that the distractor CDA in people with low WM performance resembled their high load CDA and that, conversely, the distractor condition was similar to the low load condition in people with a high WM performance. More recently, studies have even found that training in 
68 visual filtering efficiency improves working memory capacity (Li, He, Wang, Hu, \& Guo, 2017).

69 These findings suggest that people's WM performance is also strongly related to their filtering

70 efficiency, i.e., their brains ability to filter out irrelevant items.

71 Reviews of the neural origins of the CDA have concluded that the waveform is likely

72 generated by multiple sources in the brain, of which the posterior parietal cortex appears most

73 consistently mentioned (Luria et al., 2016). Posterior parietal regions have been associated with

74 the maintenance of online information across time in fMRI studies (Todd \& Marois, 2004; Xu \&

75 Chun, 2006). Considering that deficits in neural activity within fronto-parietal systems are

76 among the most consistent findings in ADHD populations (see Dickstein et al., for meta-

77 analysis), it is likely that activity in parietal regions, as partly reflected by CDA activity, could

78 play a key role in ADHD pathophysiology related to working memory problems.

79 To the best of our knowledge, only two studies thus far have investigated neural

80 correlates of storage capacity using the CDA in adults with ADHD and their peers with

81 inconsistent results. Spronk et al., (2013), found a smaller CDA for the low load condition in

82 their ADHD sample but generally concluded that there was no evidence for reliable differences

83 in neural filtering efficiency or CDA- $\Delta$ between their ADHD and comparison group. A more

84 recent study by Wiegand et al., (2016), which did not utilize a load manipulation, did find

85 differences in the CDA between individuals with ADHD and controls; showing smaller CDA

86 waves for the ADHD group which also correlated with a patient's symptom severity rating. They

87 concluded that the CDA could be a promising candidate for a neurocognitive endophenotype of

88 adult ADHD.

89 Given the paucity and conflicting nature of the research in this area to date, we tentatively

90 formulated our hypotheses as follows: 1) the ADHD group, when compared to the comparison 
91 group, will show lower CDA- $\Delta$, as operationalized by a difference measure between the CDA of

92 the low and high load condition. We expected this effect to be small since literature has shown

93 visuospatial-WM impairments in ADHD to be neither ubiquitous nor large, especially in college

94 students (Gray et al., 2016; Lambek et al., 2011; Nigg, Willcutt, Doyle, Sonuga-Barke, 2005);

95 and 2) the ADHD group will manifest decreased filtering efficiency. We expected this effect to

96 be larger since it may be indicative of a more specific and broader difficulty with selective

97 attention allowing individuals with ADHD to effectively filter out distracting information.

\section{Method}

\section{Sample}

Data reported in this manuscript were taken from a larger study investigating changes in

101

102

103

104

105

106

107

108

109

110

111

112 neural and behavioral indices after a working memory training program conducted at the [BLINDED] from 2011 to 2013 (See also; Mawjee, Woltering, \& Tannock, 2015; Mawjee, et al., 2017; Liu, Lishak, Tannock, \& Woltering, 2017). For the purpose of the current study, 27 unmedicated college students with ADHD were pair-matched with 27 peers on gender (41\% female), age (sample mean: 22 years old), and IQ (sample mean: 111). Participants with ADHD were recruited from University Student Disability Services in a major urban area via email lists and flyers. Inclusion criteria were; 1) current enrollment in a post-secondary program, 2) a previous diagnosis of ADHD, 3) registration with respective university or college Student Disability Services, which requires documented evidence previously confirmed diagnosis of ADHD (typically, but not invariably in elementary school), and 4) current symptoms consistent with diagnostic criteria for ADHD as indicated by telephone interview and meeting the criterion scores on the 6-item Adult ADHD Self-Report Scale Part A (ASRS-A). Exclusion criteria were; 
113 1) uncorrected sensory impairment or motor or perceptual handicap that would prevent the use of

114 a computer program, 2) major neurological dysfunction and psychosis or a history of concussion

115 or traumatic brain injury prior to ADHD diagnosis, and 3) limited proficiency in English

116 language. The comparison group was recruited through campus advertisements and they were

117 required to have no history or current presentation of mental health disorders. More details on

118 sample selection can be found in the supplements.

119 The study was approved by the institutional research ethics board at the University of

120 Toronto (Protocol reference: \#23977). Participants received \$20 for their participation. Informed

121 written consent was obtained from all participants prior to beginning the assessments (See copy

122 in supplements).

123 Measures

124

125

Questionnaire and behavioral task measures

126

Participants completed the Adult ADHD Self-Report Scale (ASRS v1.1; Adler et al., 2006) and the Cognitive Failures Questionnaire (CFQ; Broadbent et al., 1982; Wallace et al., 2002). The ASRS is a reliable and valid scale for evaluating current symptoms of ADHD and consists of 18 questions based on the criteria used for diagnosing ADHD in the DSM-IV-TR. For inclusion purposes, we used a modified version of the 6-item ASRS screener which was administered by telephone (for more information on this modified version and its psychometric properties, see Gray, Woltering, Mawjee, \& Tannock, 2015). The CFQ is a 25-item questionnaire with strong psychometric properties measuring self-reported failures in perception, memory, and motor function in everyday life (See, Bridger, Johnsen, \& Brasher, 2013, for recent evaluation). Four subscales were derived using factor analysis, namely the problems with 
136 Memory, Distractibility, Blunder, and (memorizing) Names (See, Wallace, Kass, \& Stanny, 137 2002).

138 To assess working memory performance using behavioral (neuropsychological)

139 measures, we administered the Cambridge Neuropsychological Testing Automated Battery

140 (CANTAB). Participants in this task needed to remember the spatial sequence of squares that

141 briefly flashed on the screen in the same order (Forwards subscale) or reverse order (Backwards

142 subscale) they appeared (Fray et al., 1996). As an estimate of general intelligence, the

143 Vocabulary and Matrix Reasoning subscales from the Wechsler Abbreviated Scale of

144 Intelligence was assessed (WASI- 2nd Ed; Wechsler, 1999).

145 Visuospatial working memory EEG task

146 Our Visual WM task was adapted from Vogel, McCollough, \& Machizawa (2005). E-

147 prime 1.2 software (Psychology Software Tools Inc.) was used to control stimulus presentation

148 and timing as well as to collect performance measures. Participants sat in front of a 17-inch VGA

149 monitor at a distance of approximately $80 \mathrm{~cm}$. Our paradigm had several phases, starting with a

150 fixation, a cue, a jitter period, a memory array, retention stage, and a test array, which are all

151 shown in Figure 1. The fixation cross appeared for $600 \mathrm{~ms}$ in the center of the screen after which

152 a cue appeared for 200 milliseconds in the form of an arrow pointing either to the left or right

153 (50\% chance) to inform participants as to which side of their visual field (i.e., left or right

154 hemifield) they should pay attention. After a 400 - $900 \mathrm{~ms}$ jitter period, a memory array

155 appeared of 2 or 4 colored shapes in both hemifields for $200 \mathrm{~ms}$ in which participants were

156 required to only memorize the colored squares in the cued hemifield. After the memory array

157 disappeared, there was a $1000 \mathrm{~ms}$ retention period during which participants needed to maintain

158 the memory array in their WM. Then, the test array appeared and participants were asked to 
159 indicate whether or not the test array was identical to the memory array by clicking one of two

160 keys on a keyboard using the index and middle fingers of their dominant hand. On half of the

161 trials, the test array was identical to the memory array, but on the other half, the color of one

162 shape on the attended side was changed. As soon as the response was made, the test array

163 disappeared and the current trial ended. If participants did not make a response within $1500 \mathrm{ms \text {, }}$

164 the trial ended automatically. The test array was shown for 500ms after which a fixation appears 165 for $1000 \mathrm{~ms}$ (for task protocol, see Figure 1).

166 The task had 3 conditions: in the low memory load condition (LL), the memory array 167 contained 2 colored squares in each hemifield; in the high memory load condition (HL), the 168 memory array contained 4 colored squares. In the distraction condition (DL), the memory array 169 contained 2 colored squares and 2 colored circles (see also Figure 1). Participants were required 170 to only memorize the squares (targets) in the attended hemifield in each condition and ignore the 171 circles (distractors) in the distraction condition. For each condition, these memory items, i.e., 172 squares (size: $0.57^{\circ} \times 0.57^{\circ}$ ) and circles (diameter: $0.57^{\circ}$ ), were randomly presented within $1735.7^{\circ} \times 8.53^{\circ}$ rectangular regions that were centered $2.86^{\circ} 4 \mathrm{~cm}$ to the right and left side of the 174 fixation cross. The color of each item was randomly chosen from 10 easily discriminable colors 175 and a given color only appeared once in the attended hemifield of memory and test arrays. The 176 task had 312 trials, with 104 trials for each condition. Trials of different conditions and of 177 different hemifield-attended sides were randomly mixed and divided within blocks. There were 17813 blocks with 24 trials in each block. At the end of each block, participants' accuracy, as well as 179 progress through the task, was shown. 
population. Instructions were presented on several subsequent screens and read aloud by a

185

186

187

188

research assistant who would verify that the participant understood the instructions. For example, participants had to repeat verbally that they were to ignore the circles in the distractor condition. Participants were also told that it was important to keep their eyes focused on the fixation cross in the center of the screen for the duration of the trial and to direct their attention, and not their eyes, to the array on the side they were instructed to attend to. Also, they were told to sit in a comfortable, relaxed position and minimize eye blinks, verbalizations, and movements as much as they could. During the practice block, research assistants were actively checking for eyemovements and would correct behavior until participants understood and complied when acting out instructions.

Participants' WM capacity (referred to as ' $\mathrm{K}$ ') was calculated from their behavioral performance. Similar to Vogel et al. (2005), we used the following formula to calculate K: K = $\mathrm{N}^{*}(\mathrm{H}-\mathrm{F})$, where $\mathrm{K}$ is visual $\mathrm{WM}$ capacity, $\mathrm{N}$ is the number of items in the memory array, $\mathrm{H}$ is the hit rate, and $\mathrm{F}$ is the false alarm rate. By subtracting $\mathrm{F}$ from $\mathrm{H}$, we can correct for guessing and obtain a more accurate capacity value. This value was calculated for the K[HL], K[LL], and $\mathrm{K}[\mathrm{DL}]$ conditions.

\section{Neural data acquisition and processing}

EEG was recorded with a 128-channel Geodesic Hydrocel Sensor Net at a $500 \mathrm{~Hz}$ sampling rate, using EGI Netstation stand-alone software (Electrical Geodesic Inc, Eugene, Oregon). Data processing was kept similar to previous literature reported on the CDA (e.g., Vogel, McCollough, \& Machizawa, 2005). EGI's Netstation software package was used to filter 
$205(.05-30 \mathrm{~Hz})$ and segment the data for correct trials $(400 \mathrm{~ms}$ before stimulus onset memory array 206 and 1000 ms post stimulus). Segments containing artifacts were removed using standard 207 automatic algorithms, including rejection of trials contaminated by blinks or eye-movements, 208 and verified manually by a research assistant blind to the study hypotheses (For more details, 209 see, Liu, Glizer, Tannock, \& Woltering, 2016). We note that blinks, vertical and horizontal eye210 movements were measured by bipolar electrodes placed above and below the left eye and at the 211 outer canthi of both eyes. On average, after removing artifacts, the control group had 71 (SD = 212 15) trials for the High Load, $83(\mathrm{SD}=14)$ trials for the Low Load, and $80(\mathrm{SD}=15)$ trials for the 213 Distractor Load. The ADHD group had $62(\mathrm{SD}=15)$ trials for the High Load, $72(\mathrm{SD}=17)$ trials 214 for the Low Load, and $66(\mathrm{SD}=17)$ trials for the Distractor Load. Trial count was not a 215 significant factor when it was added as a covariate in the main analyses.

217 further processing. Lateral-posterior sites were chosen to calculate the CDA. This selection of 218 electrodes was based on previous studies (e.g., Woodman \& Luck, 1999) and inspection of the 219 grand average waveform of all subjects. As indexed using the standard EGI system 220 nomenclature, electrode sites $52,51,59,66,61,60$, and 65 on the left hemisphere and electrode 221 sites $92,97,91,84,78,85$, and 90 on the right hemisphere were selected (see topoplot figure in 222 the supplements). First, for each condition and at the 14 electrode sites, ERPs were calculated 223 separately for left and right hemifield-attended trials. Next, ERPs on the right hemisphere 224 electrode sites were subtracted from the left sites when participants were cued to memorize the 225 right hemifield of memory arrays; and the ERPs on the left hemisphere sites were subtracted 226 from the right sites when participants were cued to the left hemifield. At this point, for each 227 condition and at each electrode site, there was a separate CDA for attended left and attended 
228 right hemifield. Then the two sides of the CDA were averaged to produce a single CDA

229 waveform. This procedure was repeated for each of the 3 conditions at each electrode site.

230 Finally, waveforms from different electrode sites were averaged to produce a final CDA

231 waveform for each condition.

232 CDA- $\Delta$ and filtering efficiency were two measures derived from the CDA waves. CDA-

$233 \Delta$ was calculated as the difference, or increase in amplitude, between the CDA amplitudes for the

234 low and high load condition (See also, Vogel et al., 2004)). To calculate the filtering efficiency

235 and quantify how efficiently participants can inhibit distractors from entering their limited WM

236 storage, we measured how individuals' CDA amplitudes in the distractors-present condition

237 resembled those CDA amplitudes in the low load (i.e., 2 items) condition rather than in the high

238 load (i.e., 4 items) condition. It was assumed that the closer the distractor CDA amplitudes are to

239 those in the low load condition, the higher the filtering efficiency is. Consistent with previous

240 literature (Vogel et al., 2005), the following formula was used to calculate this filtering

241 efficiency, $\mathrm{A}=(\mathrm{H}-\mathrm{D}) /(\mathrm{H}-\mathrm{L})$, where $\mathrm{A}$ is the filtering efficiency, and $\mathrm{H}, \mathrm{L}$, and $\mathrm{D}$ are the $\mathrm{CDA}$

242 amplitude in high load, low load, and distractor condition, respectively.

\section{Analytical Approach}

In general, standard analysis of variance analysis (ANOVA) and t-tests were applied to

245 test for differences of Group and Condition. Pearson's $r$ correlation coefficient was used to

246 investigate relationships between behavioral performance, questionnaire, and neural activity.

247 Partial eta-squared values $\left(\eta^{2}\right)$ were computed to ascertain effect size. According to Vacha-Haase

$248 \&$ Thompson (2004), partial $\eta^{2}=.01$ corresponds to a small effect, partial $\eta^{2}=.10$ corresponds

249 to a medium effect, and partial $\eta^{2}=.25$ represents a large effect. 

to perform independent t-tests along a moving window with $100 \mathrm{~ms}$ length and $50 \mathrm{~ms}$ overlap for

252 the 300-850 ms duration of the retention period. Within a group, we tested for differences

253 between the High and Low load CDA conditions. Between groups, we tested for differences in 254 filtering efficiency and the CDA- $\Delta$ (the CDA- $\Delta$ was the H-L difference measure). Previous

255 literature examining the CDA in ADHD reported different time windows for their analysis (e.g., 256 see Spronk, Vogel, \& Jonkman, 2013; Wiegand et al., 2016) but we hoped our method would 257 obtain a fine-grained, data-driven, evaluation of temporal differences. The time window in which 258 group differences were maximal would then be used to confirm group differences using an 259 ANOVA.

260 Computations were conducted with the Matlab Toolbox 'Measures of Effect Size' 261 developed by Harald Hentschk (Hentschke, Stuttgen, 2011). To remove the disproportional 262 effect of potential outliers, data were winsorized to 2 standard deviations (see, Wilcox, 2012).

263 We set our significance p-value to .05 for all analyses. Effects with p's larger than .05 and 264 smaller than .1 were treated as marginal and reported but interpreted only when in predicted 265 directions.

\section{Results}

267

\section{Behavioral \& questionnaire results}

Table 1 shows the means and standard deviations of the questionnaire and behavioral performance tasks of the ADHD group and their peers as well as the statistical tests for group differences. Results confirmed that our ADHD group reported significantly more ADHD symptomatology and everyday cognitive problems compared to their peers. Our ADHD group 
272 did not differ from their peers in their visuospatial accuracy as shown by the capacity $\mathrm{K}$ measure

273 in our change detection task. Further, no group differences were found for our standardized

274 measure of visuo-spatial WM (all $p$ 's $>.20$ ).

275

276

$<<$ INSERT TABLE 1 HERE $>>$

\section{Neural results}

278

279 280

281

282

283

284 285 286 287 288 289

290

291

292

293

Figure 2 shows the typical morphology of a CDA waveform for all three task-conditions for the ADHD and Comparison group. Statistical tests were ran during the retention period within-group between the high and low load; and between-groups for CDA- $\Delta$ and the filtering efficiency. The data showed that in the comparison group, the CDA for the low load was different from the high load condition for the period of 500-900 ms. The ADHD group showed no difference between CDA waveforms in any of the conditions. A one-way ANOVA with Group as a between-subject factor, found a significant group difference for CDA- $\Delta$ during the period of 550-700 ms, $F(1,50)=5.229, \mathrm{p}=0.027, \eta^{2}=0.0947$, with the comparison group showing a larger difference between the HL and LL compared to the ADHD group. No statistically significant group-difference effects were found for filtering efficiency at any time point (for that reason, these are not shown in figure 2).

$<<$ INSERT FIGURE 2 HERE $>>$

\section{Behavioral and Neural results}

To investigate the relationship between neural and behavioral effects between different groups, we used the neural data for which group differences were found to be maximal (e.g., the 
$294550-700 \mathrm{~ms}$ window of CDA- $\Delta$ ). The results were computed separately for the Comparison and 295 the ADHD group. Findings can be summarized by stating that none of the correlations between 296 the neural measures (e.g., CDA- $\Delta$ and filtering efficiency) and our questionnaire (e.g., ASRS, 297 CFQ) or behavioral performance measures (e.g., Capacity K and CANTAB) were statistically 298 significant.

299

300

301

302

303

304

305

306

307

308

309

310

311

312

313

314 315 316

\section{Discussion}

The present study investigated whether and how adults with ADHD differ from their peers in aspects of working memory, such as CDA- $\Delta$ and filtering efficiency, as differentiated at a neural level using a visual change detection task with distractors. The ADHD group selfreported severe impairments on measures of everyday functioning and ADHD symptomatology. Despite being severely impaired, findings showed that the ADHD group did not differ from their peers on most behavioral indices of working memory performance nor in the neural measure of filtering efficiency. However, the data did reveal a reliable pattern of lower CDA- $\Delta$ (e.g., the differentiation between high and low load) for the ADHD group.

The lack of group differences in accuracy measures of our change detection task can, in part, be explained by the nature of our sample: college students with ADHD. These subjects are relatively high-functioning adults with $\mathrm{ADHD}$, especially in their performance on standardized neuropsychological tests (See DuPaul, Weyandt, O’Dell, \& Varejo, 2009, for overview). It is possible the task was not sufficiently challenging, particularly in the low load condition as demonstrated by a skewed distribution of accuracy values. Our task, tapping into a basic working memory operation using a simple change detection task, may simply not have been demanding or complex enough to elicit differences at a behavioral level. Previous studies investigating visuospatial working memory in this college student sample using a change detection task with 
317 sequentially presented items also failed to find reliable differences in task performance measures

318 (see, Kim et al., 2014). Moreover, Gray et al., (2016) found that standardized neuropsychological

319 tests may not be ideal in differentiating college students with ADHD from their peers when

320 compared to questionnaire measures, which are likely more sensitive to impairments in relatively

321 unstructured and ongoing myriad of situations found in everyday life (For discussion, see,

322 Toplak, West, \& Stanovitch, 2013).

323

Interpretation of the neural results is less straightforward. We cannot reliably conclude

324 that processes of attentional control (e.g., selective attention) are not involved as our measure of

325 filtering efficiency requires a separation between CDAs of the high and low load condition.

326 Instead, our data showed perhaps a greater neural impairment in that the brains of individuals

327 with ADHD did not differentiate between load conditions at all. This would suggest they were

328 dedicating a similar amount of neural resources to the high and low load condition. Or perhaps

329 individuals with ADHD have basic problems with encoding stimuli rapidly (Kim et al., 2014),

330 which would account for their lack of differentiating low versus high load conditions.

331 Regardless, this pattern can be considered anomalous and it is possible that such neural

332 processing may explain impairments in visuospatial WM compared to their peers in those

333 unstructured real-life situations as their brains ultimately process such information in a less

334 efficient manner.

335 Our CDA findings appear to differ from Spronk et al., (2013) but resemble those of

336 Wiegand et al., (2016). We note that there are number of differences between the studies that

337 could explain discrepant findings. Spronk et al,, (2013) used a smaller sample of adults ( $\mathrm{n}=17)$

338 which may not have allowed for the detection of differences at a statistical level and, as Wiegand 
339 et al. (2016) noted, their task had a maximal set size of three items which may not have been

340 enough to discern load differences optimally.

While it would be premature to characterize the CDA as an endophenotype of ADHD

342 (Biomarkers Definition Working Group, 2001; de Geus, 2010) and we do caution clinical

343 implications, our study did suggest neural differences in the brain's processing of working

344 memory. Our findings fit in a larger body of literature that has found evidence of anomalous

345 processing at a neural level in adult ADHD of information in working memory with EEG (e.g.,

346 Kim et al., 2014; Missonier et al., 2013; Lenartowicz et al., 2014) and other neural methods

347 (Ehlis et al., 2008; Schweitzer et al., 2000; Valera et al., 2005).

348 We also want to point out limitations and alternative explanations/potential moderators of 349 our effects. First, the task was not entirely self-paced (i.e., participants had to be continuously 350 engaged and ready for the next trial) and different conditions were presented in a random manner 351 mixed within blocks. Furthermore, there was a jitter period which also made the timing of the 352 onset of the stimulus unpredictable. As such, it is possible the neural effects could also be attributed to difficulties with task switching and readiness or the timed allocation of attentional

354 355

357

resources (Cubillo et al, 2010; Dockstader et al., 2008). Second, an emerging literature is suggesting individuals with ADHD have difficulty with color processing making color a less salient feature, which could also explain our lack of effects in the ADHD group as they were asked to detect color changes (Banaschewski et al., 2006; Kim, Chen, \& Tannock, 2014).

Finally, the lack of statistically significant correlations between our behavioral and CDA neural findings, in particular for our comparison group, fails to replicate basic findings in the literature. Due to the nature of our population, we were forced to keep trial counts per condition low, or at least lower than they have been in most of the literature in typically developing populations. It is 
362 possible that this could have attributed to these non-significant effects. Alternatively, we can also

363 not exclude the possibility that the neural activity we captured during this task may be capturing

364 more general processes involved in the execution, but not reflected in the performance, of the

365 task. The CDA may be a less reliable index of visual WM capacity in individuals with impaired

366 attention and WM functions, such as ADHD patients (Wiegand et al., 2016), but also other

367 groups, such as healthy older adults (Jost et al., 2011; Sander et al., 2011; Wiegand et al., 2018).

368

369 Conclusions

370 Notwithstanding the aforementioned limitations, the present study contributes to an

371 emerging literature investigating working memory in subjects with ADHD from a neural

372 perspective. Our findings suggest that the brains of individuals with ADHD process the

373 maintenance of information differently from their healthy peers. More research would be needed

374 to confirm our findings and further specify what this means for the characterization of this

375 debilitating condition.

376

377 References

378

379 Awh E, Vogel E, Oh S. 2006. Interactions between attention and working memory. Neuroscience 380 139: 201-208.

381

382 Baddeley A. 2010. Working memory. Current biology 20: R136-R140.

383 
384 Banaschewski T, Ruppert S, Tannock R, Albrecht B, Becker A, Uebel H, Sergeant JA, 385 Rothenberger A. 2006. Colour perception in ADHD. Journal of Child Psychology and 386 Psychiatry 47: 568-572.

387

388 Biomarkers Definition Working Group. 2001. Biomarkers and surrogate endpoints: Preferred 389 definitions and conceptual framework. Clinical Pharmacology and Therapeutics 69: 89-95. 390

391 Bridger RS, Johnsen SÅK, Brasher K. 2013. Psychometric properties of the cognitive failures 392 questionnaire. Ergonomics 56: 1515-1524.

393

394 Broadbent DE, Cooper PF, FitzGerald P, Parkes KR. 1982. The cognitive failures questionnaire 395 (CFQ) and its correlates. British Journal of Clinical Psychology 21: 1-16.

396

397 Bunge SA, Klingberg T, Jacobsen RB, Gabrieli JD. 2000. A resource model of the neural basis 398 of executive working memory. Proceedings of the National Academy of Sciences 97: 3573-3578.

399

400 Chamberlain SR, Robbins TW, Winder-Rhodes S, Müller U, Sahakian BJ, Blackwell AD, 401 Barnett JH. 2011. Translational approaches to frontostriatal dysfunction in attention402 deficit/hyperactivity disorder using a computerized neuropsychological battery. Biological 403 Psychiatry 69: 1192-1203. 
405 Cortese S, Kelly C, Chabernaud C, Proal E, Di Martino A, Milham MP, Castellanos FX. 2012.

406 Toward systems neuroscience of ADHD: A meta-analysis of 55 fMRI studies. American Journal 407 of Psychiatry 169: 1038-1055.

408

409 Cowan N. 2001. The magical number 4 in short-term memory: A reconsideration of mental 410 storage capacity. Behavioral and Brain Sciences 24: 154-176.

411

412 Cowan N, Elliott EM, Saults JS, Morey CC, Mattox S, Hismjatullina A, Conway AR. 2005. On 413 the capacity of attention: Its estimation and its role in working memory and cognitive aptitudes. 414 Cognitive Psychology 51: 42-100.

415

416 Cubillo A, Halari R, Ecker C, Giampietro V, Taylor E, Rubia K. 2010. Reduced activation and 417 inter-regional functional connectivity of fronto-striatal networks in adults with childhood 418 Attention-Deficit Hyperactivity Disorder (ADHD) and persisting symptoms during tasks of 419 motor inhibition and cognitive switching. Journal of Psychiatric Research 44: 629-639.

420

421 de Geus EJ. 2010. From genotype to EEG endophenotype: A route for post-genomic 422 understanding of complex psychiatric disease? Genome Medicine 2, 63.

423

424 de Graaf R, Kessler RC, Fayyad J, ten Have M, Alonso J, Angermeyer M, Borges G, 425 Demyttenaere K, Gasquet I, de Girolamo G, Haro JM, Jin R, Karam EG, Ormel J, Posada-Villa 426 J. 2008. The prevalence and effects of adult attention-deficit/hyperactivity disorder (ADHD) on 
427 the performance of workers: Results from the WHO World Mental Health Survey Initiative.

428 Occupational and Environmental Medicine 65: 835-842.

429

430 Diamantopoulou S, Poom L, Klaver P, Talsma D. 2011. Visual working memory capacity and

431 stimulus categories: a behavioral and electrophysiological investigation. Experimental Brain

432 Research 209: 501-513.

433

434 Dickstein SG. Bannon K, Castellanos FX, Milham MP. 2006. The neural correlates of attention 435 deficit hyperactivity disorder: An ALE meta-analysis. Journal of Child Psychology and 436 Psychiatry, 47: 1051-1062.

437

438

Dockstader C, Gaetz W, Cheyne D, Wang F, Castellanos FX, Tannock R. 2008. MEG event-

439 related desynchronization and synchronization deficits during basic somatosensory processing in individuals with ADHD. Behavioral and Brain Functions 4, 8.

441

442

DuPaul GJ, Weyandt LL, O'Dell SM, Varejao M. 2009. College students with ADHD: Current

443 status and future directions. Journal of Attention Disorders 13: 234-250.

444

445 Ehlis AC, Bähne CG, Jacob CP, Herrmann MJ, Fallgatter AJ. 2008. Reduced lateral prefrontal

446 activation in adult patients with attention-deficit/hyperactivity disorder (ADHD) during a

447 working memory task: a functional near-infrared spectroscopy (fNIRS) study. Journal of

448 Psychiatric Research 42: 1060-1067. 
450 Fray P, Robbins T, Sahakian B. 1996. Neuropsychiatric applications of CANTAB. International 451 Journal of Geriatric Psychiatry 11: 329-336.

452

453 Fried R, Chan J, Feinberg L, Pope A, Woodworth KY, Faraone SV, Biederman J. 2016. Clinical 454 correlates of working memory deficits in youth with and without ADHD: A controlled study.

455 Journal of Clinical and Experimental Neuropsychology 38: 487-496.

456

457 Gray SA, Fettes P, Woltering S, Mawjee K, Tannock R. 2016. Symptom manifestation and 458 impairments in college students with ADHD. Journal of Learning Disabilities 49: 616-630.

459

460 Gray S, Woltering S, Mawjee K, Tannock R. 2014. The Adult ADHD Self-Report Scale 461 (ASRS): Utility in college students with attention-deficit/hyperactivity disorder. PeerJ 2: e324.

462

463 Hentschke H, Stüttgen MC. 2011. Computation of measures of effect size for neuroscience data 464 sets. European Journal of Neuroscience 34: 1887-1894.

465

466 Hervey AS, Epstein JN, Curry JF. 2004. Neuropsychology of adults with attention-

467 deficit/hyperactivity disorder: A meta-analytic review. Neuropsychology 18: 485-503.

468

469 Jost K, Bryck RL, Vogel EK, Mayr U. 2010. Are old adults just like low working memory young 470 adults? Filtering efficiency and age differences in visual working memory. Cerebral cortex 21: $471 \quad 1147-1154$

472 
473 Kim S, Chen S, Tannock R. 2014. Visual function and color vision in adults with Attention-

474 Deficit/Hyperactivity Disorder. Journal of Optometry 7: 22-36.

475

476 Kim S, Liu Z, Glizer D, Tannock R, Woltering S. 2014. Adult ADHD and working memory:

477 Neural evidence of impaired encoding. Clinical Neurophysiology 125: 1596-1603.

478

479 Kuo BC, Stokes MG, Nobre AC. 2012. Attention modulates maintenance of representations in 480 visual short-term memory. Journal of Cognitive Neuroscience 24: 51-60.

481

482 Kundu B, Sutterer DW, Emrich SM, Postle BR. 2013. Strengthened effective connectivity 483 underlies transfer of working memory training to tests of short-term memory and attention. 484 Journal of Neuroscience 33: 8705-8715.

485

486

Lambek R, Tannock R, Dalsgaard S, Trillingsgaard A, Damm D, Thomsen PH. 2011. Executive 487 dysfunction in school-age children with ADHD. Journal of Attention Disorders 15: 646-655.

488

489

Lenartowicz A, Delorme A, Walshaw PD, Cho AL, Bilder RM, McGough JJ, Loo SK. 2014.

490 Electroencephalography correlates of spatial working memory deficits in attention-

491 deficit/hyperactivity disorder: Vigilance, encoding, and maintenance. Journal of Neuroscience 492 34: 1171-1182.

493

494 Li CH, He X, Wang YJ, Hu Z, Guo CY. 2017. Visual working memory capacity can be 495 increased by training on distractor filtering efficiency. Frontiers in Psychology 8: 196. 
497 Liu ZX, Glizer D, Tannock R, Woltering S. 2016. EEG alpha power during maintenance of 498 information in working memory in adults with ADHD and its plasticity due to working memory

499 training: A randomized controlled trial. Clinical Neurophysiology 127: 1307-1320.

500

501 Liu ZX, Lishak V, Tannock R, Woltering S. 2017. Effects of working memory training on neural 502 correlates of Go/Nogo response control in adults with ADHD: A randomized controlled trial.

503 Neuropsychologia 95: 54-72.

504

505 Luria R, Balaban H, Awh E, Vogel EK. 2016. The contralateral delay activity as a neural 506 measure of visual working memory. Neuroscience \& Biobehavioral Reviews 62: 100-108.

507

508 Martinussen R, Hayden J, Hogg-Johnson S, Tannock R. 2005. A meta-analysis of working 509 memory impairments in children with attention-deficit/hyperactivity disorder. Journal of the 510 American Academy of Child \& Adolescent Psychiatry 44: 377-384.

511

512 Mawjee K, Woltering S, Lai N, Gotlieb H, Kronitz R, Tannock R. 2017. Working memory

513 training in ADHD: Controlling for engagement, motivation, and expectancy of improvement

514 (pilot study). Journal of Attention Disorders 21: 956-968.

515

516 Mawjee K, Woltering S, Tannock R. 2015. Working memory training in post-secondary students 517 with ADHD: A randomized controlled study. PloS one 10: e0137173. 
519 McCollough AW, Machizawa MG, Vogel EK. 2007. Electrophysiological measures of

520 maintaining representations in visual working memory. Cortex 43: 77-94.

521

522 Missonnier P, Hasler R, Perroud N, Herrmann FR, Millet P, Richiardi J, Malafosse A,

523 Giannakopoulos P, Baud P. 2013. EEG anomalies in adult ADHD subjects performing a working 524 memory task. Neuroscience 241: 135-146.

525

526 Miyake A, Shah P. 1999. Models of working memory: Mechanisms of active maintenance and 527 executive control. Cambridge University Press.

528

529 Nigg JT, Willcutt EG, Doyle AE, Sonuga-Barke EJ. 2005. Causal heterogeneity in attention530 deficit/hyperactivity disorder: Do we need neuropsychologically impaired subtypes?. Biological 531 Psychiatry 57: 1224-1230.

532

533 Polanczyk G, Jensen P. 2008. Epidemiologic considerations in attention deficit hyperactivity

534 disorder: A review and update. Child and Adolescent Psychiatric Clinics of North America 17: $535 \quad 245-260$.

536

537 Sander MC, Werkle-Bergner M, Lindenberger U. 2011. Contralateral delay activity reveals life538 span age differences in top-down modulation of working memory contents. Cerebral Cortex 21: 539 2809-2819.

540 
541 Schweitzer JB, Faber TL, Grafton ST, Tune LE, Hoffman JM, Kilts CD. 2000. Alterations in the

542 functional anatomy of working memory in adult attention deficit hyperactivity disorder.

543 American Journal of Psychiatry 157: 278-280.

544

545 Shipstead Z, Lindsey DR, Marshall RL, Engle RW. 2014. The mechanisms of working memory

546 capacity: Primary memory, secondary memory, and attention control. Journal of Memory and

547 Language 72: 116-141.

548

549 Spronk M, Vogel EK, Jonkman LM. 2013. No behavioral or ERP evidence for a developmental 550 lag in visual working memory capacity or filtering in adolescents and adults with ADHD. PloS 551 one 8: e62673.

552

553 Todd JJ, Marois R. 2004. Capacity limit of visual short-term memory in human posterior parietal 554 cortex. Nature 428: 751.

555

556 Toplak ME, West RF, Stanovich KE. 2013. Practitioner Review: Do performance-based 557 measures and ratings of executive function assess the same construct?. Journal of Child 558 Psychology and Psychiatry 54: 131-143.

559

560 Uchida M, Spencer TJ, Faraone SV, Biederman J. 2018. Adult outcome of ADHD: an overview 561 of results from the MGH longitudinal family studies of pediatrically and psychiatrically referred 562 youth with and without ADHD of both sexes. Journal of Attention Disorders 22: 523-534. 
564 Vacha-Haase T, Thompson B. 2004. How to estimate and interpret various effect sizes. Journal 565 of Counseling Psychology 51: 473-481.

566

567 Valera EM, Faraone SV, Biederman J, Poldrack RA, Seidman LJ. 2005. Functional

568 neuroanatomy of working memory in adults with attention-deficit/hyperactivity disorder.

569 Biological Psychiatry 57: 439-447.

570

571 Vogel EK, McCollough AW, Machizawa MG. 2005. Neural measures reveal individual

572 differences in controlling access to working memory. Nature 438: 500-503.

573

574 Vogel EK, Machizawa MG. 2004. Neural activity predicts individual differences in visual 575 working memory capacity. Nature 428: 748-751.

576

577 Wallace JC, Kass SJ, Stanny CJ. 2002. The cognitive failures questionnaire revisited: dimensions 578 and correlates. The Journal of General Psychology 29: 238-256.

579

580 Wechsler D. 1999. Wechsler Abbreviated Scale of Intelligence. Harcourt Brace, New 581 York, NY.

582

583 Wiegand I, Hennig-Fast K, Kilian B, Müller HJ, Töllner T, Möller HJ, Engel RR, Finke K. 2016. 584 EEG correlates of visual short-term memory as neuro-cognitive endophenotypes of ADHD. 585 Neuropsychologia 85: 91-99. 
587 Wiegand I, Lauritzen MJ, Osler M, Mortensen EL, Rostrup E, Rask L, ... Petersen A. 2018. EEG

588 correlates of visual short-term memory in older age vary with adult lifespan cognitive

589 development. Neurobiology of aging 62: 210-220.

590

591 Wilcox RR. 2012. Introduction to robust estimation and hypothesis testing (Third edition).

592 Boston: Academic Press.

593

594 Woodman GF, Luck SJ. 1999. Electrophysiological measurement of rapid shifts of attention

595 during visual search. Nature 400: 867-869.

596

597 Xu Y, Chun MM. 2006. Dissociable neural mechanisms supporting visual short-term memory

598 for objects. Nature, 440: 91. 
Figure 1 (on next page)

Simplified flowchart of the visuospatial change detection task with distractors 


\section{Figure 2}

CDA waveforms for the low load (blue), high load (red), and distractor condition (yellow) for the Comparison (top panel) and ADHD group (bottom panel).

Stimulus onset starts at $0 \mathrm{~ms}$ for $200 \mathrm{~ms}$. The bars at the top and bottom indicate withinGroup Condition differences between the high (HL) and low load (LL) CDA. The two bars in the middle test Group differences for 'CDA- $\Delta$ ' (difference measure of $\mathrm{HL}-\mathrm{LL}$ ) and 'Filtering Efficiency'. Bars tested differences using a $100 \mathrm{~ms}$ time window with $50 \mathrm{~ms}$ overlap with the color red representing: ${ }^{*} p<.01$; and orange: $* p<.05$. 


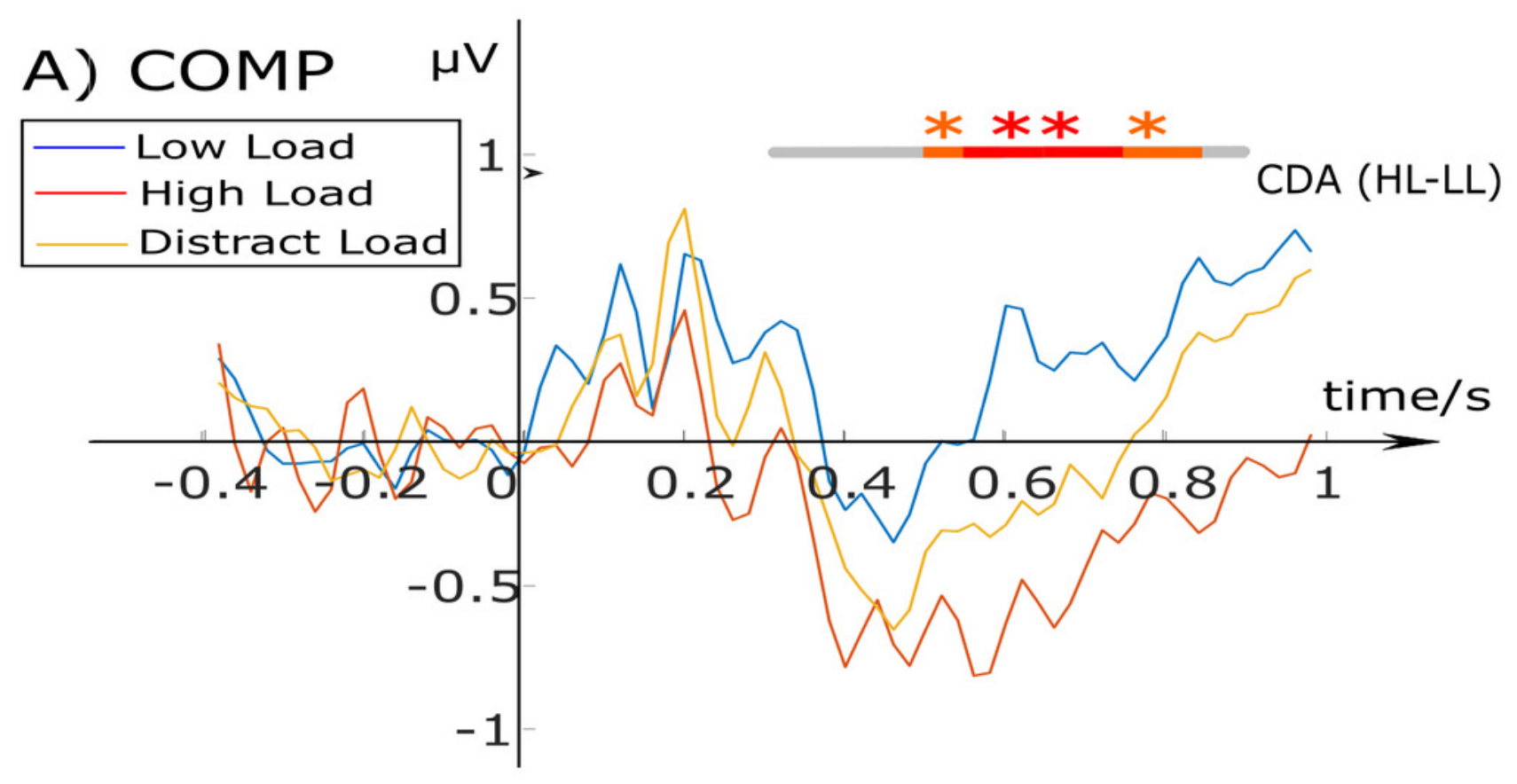

Filtering Efficiency CDA- $\triangle$

*

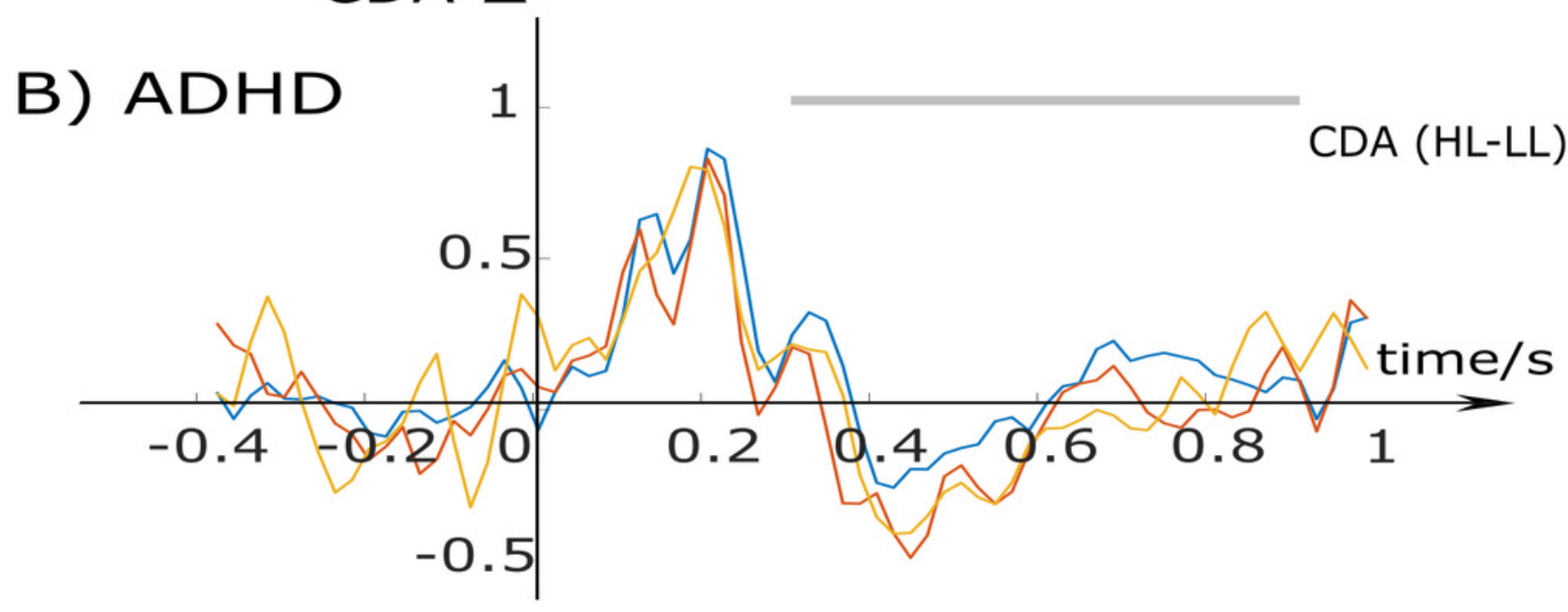




\section{Table $\mathbf{1}$ (on next page)}

Descriptives for questionnaire and behavioral measurements and group differences

$* p<.05 ; * * p<.01 ; * * *<<.001$. 
1 Table 1. Descriptive and group differences for questionnaire and behavioral measurements 2

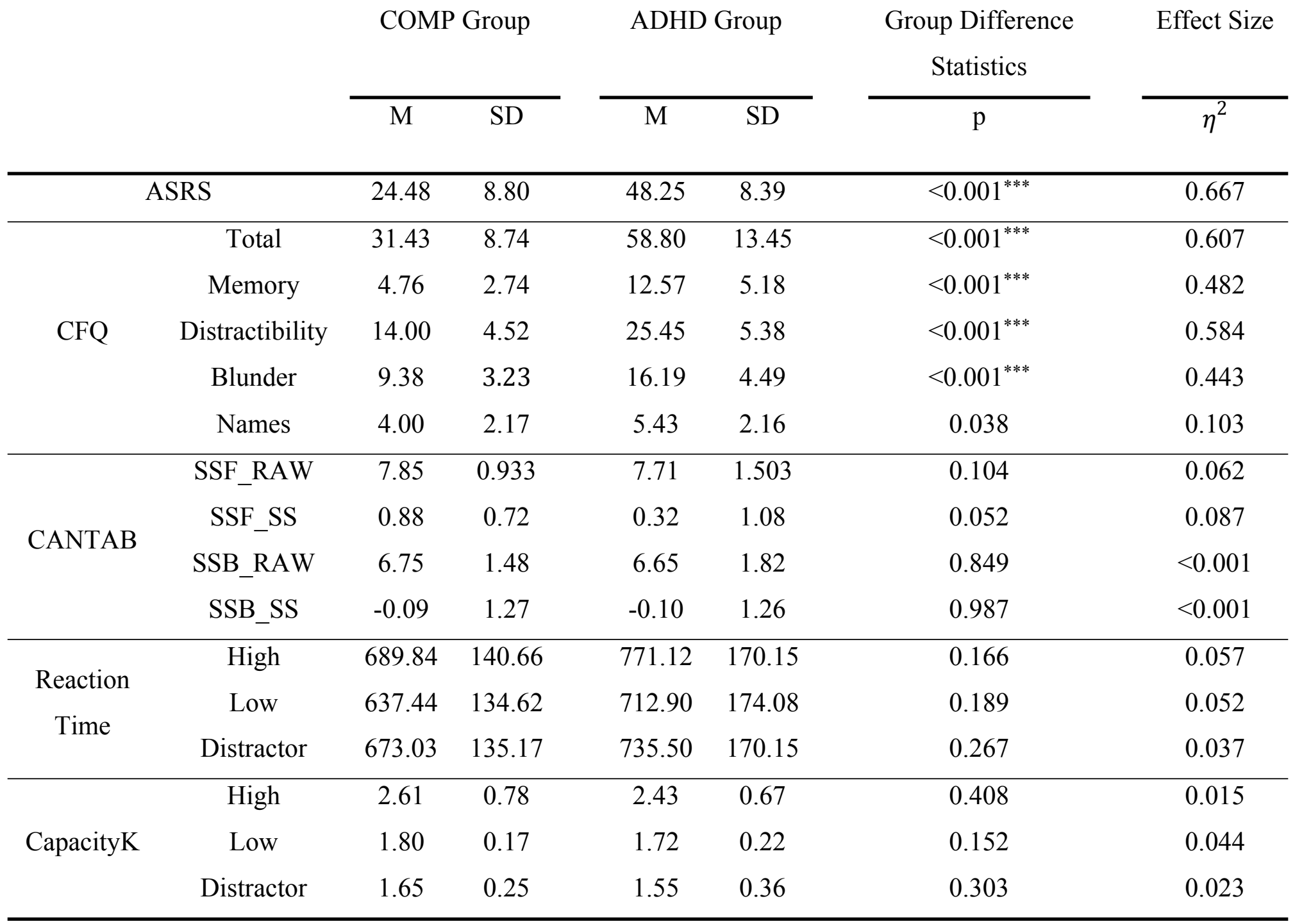

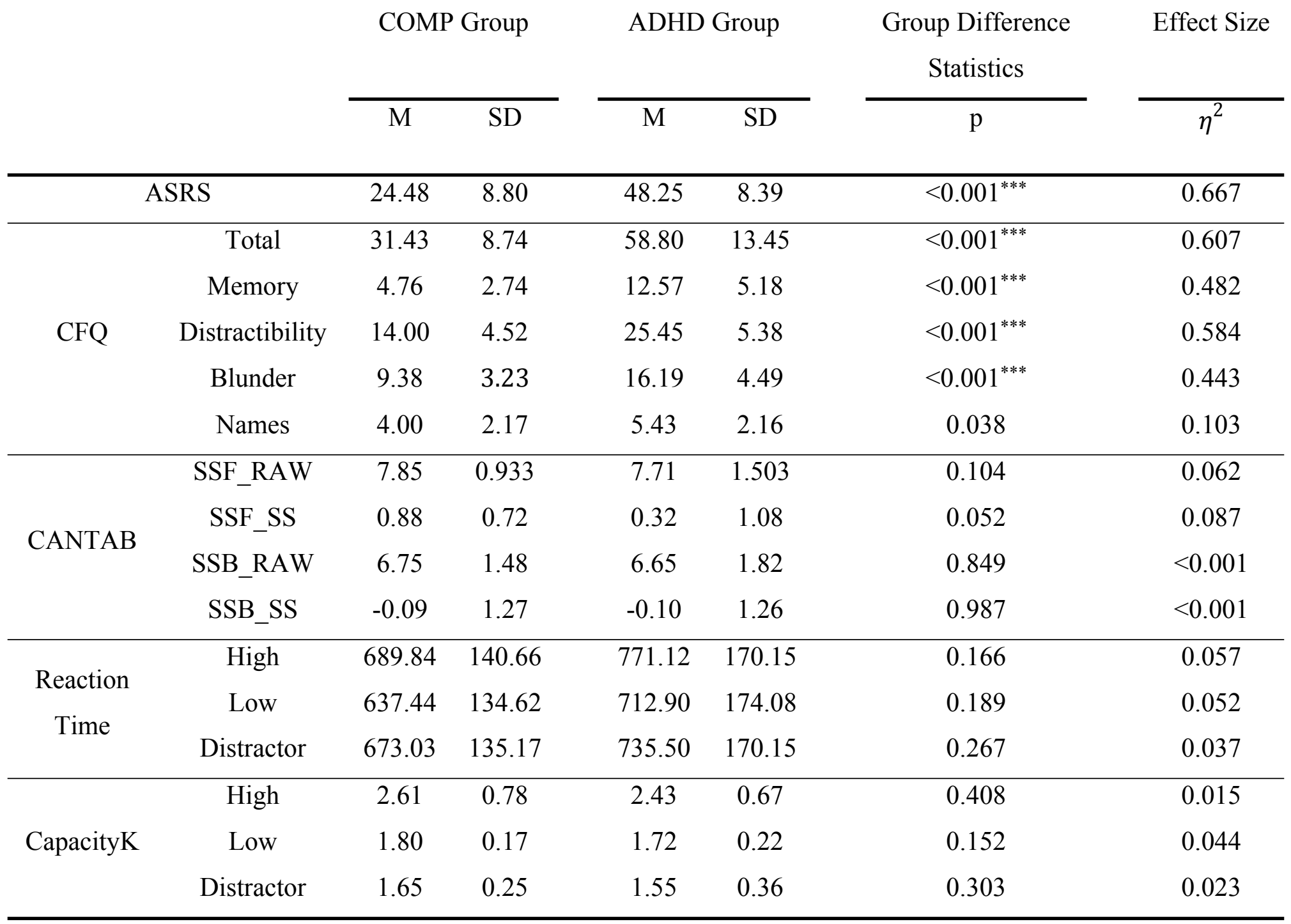

Effect Size 